\title{
Engineering interactions and anyon statistics by multicolor lattice-depth modulations
}

\author{
Lorenzo Cardarelli, Sebastian Greschner, and Luis Santos \\ Institut für Theoretische Physik, Leibniz Universität Hannover, Appelstrasse 2, DE-30167 Hannover, Germany
}

(Received 4 May 2016; revised manuscript received 13 July 2016; published 11 August 2016)

\begin{abstract}
We show that a multicolor modulation of the depth of an optical lattice allows for a flexible independent control of correlated hopping, occupation-dependent gauge fields, effective on-site interactions without Feshbach resonances, and nearest-neighbor interactions. As a result, the lattice-depth modulation opens the possibility of engineering with minimal experimental complexity a broad class of lattice models in current experiments with ultracold atoms, including Hubbard models with correlated hopping, peculiar extended models, and twocomponent anyon-Hubbard models.
\end{abstract}

DOI: 10.1103/PhysRevA.94.023615

\section{INTRODUCTION}

Floquet engineering - the averaging of fast periodic modulations to obtain an effective time-independent system-is a ubiquitous tool for the manipulation and probing of various systems, ranging from NMR probes in solid-state physics to atom-light interactions or Raman-dressed states [1]. In recent years, Floquet techniques have established themselves as a toolbox for the creation of novel Hamiltonians for ultracold atoms in optical lattices, including lattice shaking [2-8], Raman-assisted hopping [9-12], and modulated interactions [13-18].

A major reason for the interest on Floquet techniques lies in the possibility of engineering gauge fields, i.e., complex hopping rates, for neutral atoms in optical lattices [7]. Most relevantly, synthetic magnetic fields have been created in the last years using Raman-assisted hopping [9-12]. Interestingly, various Floquet techniques have been recently proposed for the creation of occupation-dependent gauge fields (ODGs) [19-22], in which the phase of the hopping depends on the site occupation. Under proper conditions, one-dimensional (1D) models with ODGs may be mapped into an anyon-Hubbard model (AHM) [19-22], in which the exchange statistics of the atoms may be externally modified. The 1D AHM presents a wealth of new physics, including statistically induced transitions [20], novel superfluid phases [21], smooth fermionization [22], asymmetric momentum distributions [23,24], and intriguing dynamics [25-27]. The atomic back-action on the synthetic gauge field given by ODGs could pave a way for the realization of dynamical gauge fields [28,29], and leads to interesting physics, such as chiral solitons in Bose-Einstein condensates [30] or density-flux interplay in two-dimensional lattices [31].

In this paper, we propose a method based on the multicolor modulation of the depth of a tilted optical lattice. As shown by Ma et al. [32] lattice-depth modulations may be employed to assist different occupation-dependent hopping processes for sufficiently strong interactions. We show for the particular case of two-component fermions that a three-color modulation (3CM) of the lattice depth may be employed to achieve a separate flexible control of correlated hopping, ODGs, effective on-site interactions without the need of Feshbach resonances, and nearest-neighbor (NN) interactions. As a result, $3 \mathrm{CM}$ allows with a minimal experimental complexity for engineering a broad class of lattice Hamiltonians using

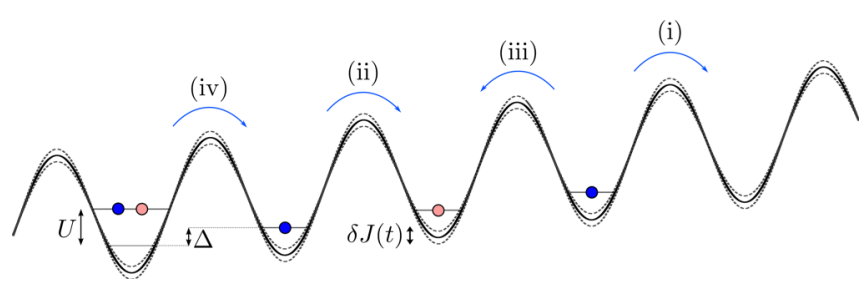

FIG. 1. Sketch of the lattice setup and the relevant hopping processes.

ultracold atoms, including Hubbard models with correlated hopping, peculiar extended models, and two-component AHMs, the basic properties of which we analyze as well.

This work is organized as follows. In Sec. II we introduce the multicolor modulation method and derive the effective Hamiltonian that describes the system. Section III deals with the investigation of the nonequilibrium dynamics, both for sudden quenches and for a quasiadiabatic ramping, that validates the effective Hamiltonian in comparison to the full time-dependent Hamiltonian. Section IV presents a detailed analysis of the physics that can be explored with the effective Hamiltonian. Finally, Sec. V offers a summary of the work and the outlook.

\section{EFFECTIVE HAMILTONIAN}

In this section we present a method that allows us to realize in a one-dimensional optical lattice a Fermi-Hubbard model with a density-dependent Peierls phase, and that offers a flexible experimental control on the different terms of the Hamiltonian. We obtain an effective time-independent Hamiltonian that fully characterizes the ground-state physics and the dynamics of the system.

\section{A. Three-color modulation on the tilted lattice}

We consider a balanced two-component $(\sigma=\uparrow, \downarrow)$ Fermi gas in an optical lattice (equal for both components), the depth of which is modulated in time, $V(t)=V_{0}+\delta V(t)$, with $\delta V \ll V_{0}$. We choose two-component fermions for simplicity, but similar ideas may be applied to bosons, and multicomponent fermions. In the tight-binding regime, the hopping rate is $\frac{J(s)}{E_{\text {rec }}}=\frac{4}{\sqrt{\pi}} s^{3 / 4} \exp (-2 \sqrt{s})$ [33], where $s=$ $V / E_{\mathrm{rec}}=s_{0}+\delta s(t)$, with $E_{\mathrm{rec}}$ the recoil energy associated 
to the laser that creates the lattice. Since $\delta s \ll s_{0}$, then $J(t)=$ $J_{0}+\delta J(t)$, where $J_{0}=J\left(s_{0}\right)$, and $\frac{\delta J(t)}{J_{0}}=\left(\frac{3}{4}-\sqrt{s_{0}}\right) \frac{\delta s(t)}{s_{0}}$, and hence the lattice modulation directly maps into a modulation of the hopping rate. We assume a tilted lattice, with an energy shift $\Delta$ between neighboring sites (Fig. 1). The system is then described by the Fermi-Hubbard Hamiltonian:

$$
\mathcal{H}(t)=-J(t) \sum_{j, \sigma}\left[c_{j+1, \sigma}^{\dagger} c_{j, \sigma}+\text { H.c. }\right]+U \mathcal{H}_{\text {int }}+\Delta \mathcal{H}_{\text {tilt }},
$$

where $c_{j, \sigma}$ is the annihilation operator of a fermion with spin $\sigma$ at site $j, U$ characterizes the on-site interactions, $\mathcal{H}_{\text {int }}=\sum_{j} n_{j, \uparrow} n_{j, \downarrow}$, and $\mathcal{H}_{\text {tilt }}=\sum_{j, \sigma} j n_{j, \sigma}$. Note that four different hopping processes are possible (as sketched in Fig. 1).

(i) A single atom hops to an empty site to its right leading to an energy shift $\Delta E_{1}=\Delta$.

(ii) An atom with spin $\sigma$, initially alone at a given site, tunnels to the site at its right already occupied by a single atom with $\bar{\sigma} \neq \sigma$, resulting in a shift $\Delta E_{2}=\Delta+U$.

(iii) The same event as (ii) but the hopping is to the left: in this case $\Delta E_{3}=U-\Delta$.

(iv) An atom of component $\sigma$ sharing a site with a $\bar{\sigma}$ atom tunnels into the site at its right already occupied by a single atom with $\bar{\sigma}$ leading to an energy shift $\Delta E_{4}=\Delta$. Note that processes (iv) and (i) are resonant.

We assume that $J(t) \ll \Delta,|\Delta \pm U|$, and hence direct hopping is negligible. However, a periodic modulation of $\delta J(t)$ leads to assisted hopping if the modulation frequency matches the energy shift associated to the hopping process [32]. Crucially, processes (i), (ii) and (iii) are characterized by different energy shifts (by twos, typically separated by several $\mathrm{kHz}$, see below), and hence the different hopping processes may be laser assisted separately. The key point of our proposal is to address them separately but simultaneously using a $3 \mathrm{CM}$ of the laser intensity: $\delta V(t)=\sum_{s=1,2,3} \delta V_{s} \cos \left(\omega_{s} t+\phi_{s}\right)$, which, as mentioned above, translates into an equivalent modulation of the hopping, $\delta J(t)=\sum_{s} \delta J_{s} \cos \left(\omega_{s} t+\phi_{s}\right)$. Each component of the modulation has an amplitude $\delta J_{s}$ and a phase shift $\delta \phi_{s}$, which may be independently controlled. The frequencies $\omega_{1}=\Delta, \omega_{2}=\Delta+U-\tilde{U}$, and $\omega_{3}=-\Delta+U-\tilde{U}$, with $|\tilde{U}| \ll U$ are chosen (quasi-)resonant to the hopping processes (i) [and hence also to (iv)], (ii), and (iii), respectively.

\section{B. Time-independent Hamiltonian}

The relevant physics of the time-dependent Hamiltonian just introduced is adequately described by an effective timeindependent Hamiltonian that we derive and comment upon hereafter. In the interaction picture, $\tilde{\mathcal{H}}=\mathcal{U}^{\dagger} \mathcal{H} \mathcal{U}$, with $\mathcal{U}=$ $\exp \left[-i t\left(\Delta \mathcal{H}_{\text {tilt }}+U \mathcal{H}_{\text {int }}\right)\right]$ :

$$
\tilde{\mathcal{H}}(t)=-J(t) \sum_{j, \sigma}\left[c_{j+1, \sigma}^{\dagger} e^{i t\left[\Delta+U\left(n_{j+1, \bar{\sigma}}-n_{j, \bar{\sigma}}\right)\right]} c_{j, \sigma}+\text { H.c. }\right] .
$$

The 3CM introduces oscillating terms $e^{ \pm i\left(\omega_{s} \pm \Delta E_{s^{\prime}}\right) t}$. For $J_{0} \ll$ $|\Delta-U|, U$ the fast-oscillating terms average to zero [rotating wave approximation (RWA)], and only quasiresonant terms remain (see Appendix B for an alternative derivation). As a result, processes (i) [and (iv)], (ii), and (iii) present an effective hopping rate $\frac{\delta J_{s}}{2} e^{i \phi_{s}}$, with $s=1,2$, and 3 , respectively. We consider below the particular case with $\delta J_{2,3}=\beta \delta J_{1}, \phi_{1}=$ $0, \phi_{2,3}=\phi$. Undoing the interaction picture we obtain the effective time-independent Hamiltonian:

$$
\mathcal{H}_{\mathrm{eff}}=-\frac{\delta J_{1}}{2} \sum_{j, \sigma} c_{j+1, \sigma}^{\dagger} F\left[\left|n_{J+1, \bar{\sigma}}-n_{\bar{\sigma}, j}\right|\right] c_{j, \sigma}+\tilde{U} \mathcal{H}_{\mathrm{int}},
$$

where $F[0]=1$ and $F[1]=\beta e^{i \phi}$. The $3 \mathrm{CM}$ provides remarkable control possibilities. Both the amplitude and the phase of the hopping rate of the $\sigma$ component depend on the site occupation of the $\bar{\sigma}$ component. As shown below, this may be employed to realize ODGs. Moreover, the detuning $\tilde{U}$ results in an effective on-site interaction, allowing for controlling interactions even in those systems where Feshbach resonances are not available. This is in particular the case of alkaline-earth fermions in the lowest ${ }^{1} S_{0}$ state [34]. Since 3CM may be also used with multicomponent fermions, this opens a way of controlling the properties of SU(N) fermions [34].

Although for $J(t) \ll \Delta,|\Delta \pm U|$ direct hopping is energetically forbidden, virtual hopping may induce effective interactions between NN sites (see Appendix B) of the form

$$
\begin{aligned}
\mathcal{H}_{\mathrm{NN}}= & \sum_{\langle i, j\rangle}\left[\frac{2 J_{0}^{2}}{\Delta+U} P_{i}^{0} P_{j}^{2}-\frac{2 J_{0}^{2}}{\Delta-U} P_{i}^{2} P_{j}^{0}\right. \\
& +\frac{J_{0}^{2}}{\Delta}\left[\left(1-n_{i}\right) P_{j}^{1}-P_{i}^{1}\left(1-n_{j}\right)\right] \\
& \left.+\frac{2 U J_{0}^{2}}{\Delta^{2}-U^{2}}\left(P_{i}^{1 \uparrow} P_{j}^{1 \downarrow}+P_{i}^{1 \downarrow} P_{j}^{1 \uparrow}-S_{i}^{+} S_{j}^{-}-S_{i}^{-} S_{j}^{+}\right)\right],
\end{aligned}
$$

where $S_{i}^{+}=c_{i, \uparrow}^{\dagger} c_{i, \downarrow}$ and $S_{i}^{-}=c_{i, \downarrow}^{\dagger} c_{i, \uparrow}$ are spin operators, $n_{i}=$ $n_{i, \downarrow}+n_{i, \uparrow}$, and we introduce the projector of two particles per site $P_{i}^{2}=n_{i, \downarrow} n_{i, \uparrow}$, zero particles $P_{i}^{0}=\left(1-n_{i, \downarrow}\right)\left(1-n_{i, \uparrow}\right)$, and a single particle $P_{i}^{1 \sigma}=\left(1-n_{i, \bar{\sigma}}\right) n_{i, \sigma}$, and $P_{i}^{1}=P_{i}^{1 \downarrow}+$ $P_{i}^{1 \uparrow}$. The peculiar NN interactions depend on $J_{0}^{2} / \Delta$ and $J_{0}^{2} /(U \pm \Delta)$, whereas the effective hopping is given by $\delta J_{i}$. Hence they may be separately controlled. For sufficiently small $J_{0} \ll \Delta,|U \pm \Delta|$ we may neglect $\mathcal{H}_{\mathrm{NN}}$. However, as shown below, $\mathcal{H}_{\mathrm{NN}}$ opens additional interesting possibilities.

The rotating wave approximation requirements necessary for the three-color modulation are readily achievable experimentally. As an example, let us consider the Ytterbium isotope ${ }^{173} \mathrm{Yb}$, with scattering length $a_{0}=199.4 a_{B}$, a lattice with spacing $d=380 \mathrm{~nm}[11,35]$ and depth $s_{0}=6.9$, tilted in gravity. By virtue of the tight confinement, the on-site interaction may be quantified in harmonic approximation. In this case, one has a hopping rate $J_{0} / h=100 \mathrm{~Hz}$, and $U=23 J_{0}, \Delta=16 J_{0}$ (for a lattice oriented along the gravity, largest tilting), $|\Delta-U|=7 J_{0}$, well within the RWA requirements. For $\delta J / J_{0}=0.2$, the typical effective-tunneling time is $\tau=\hbar / \delta J \simeq 8 \mathrm{~ms}$.

\section{NONEQUILIBRIUM DYNAMICS}

We employ numerical simulations to examine the timedependent properties of the model of Eq. (1) and the effective description of Eq. (3), including the second-order corrections of Eq. (4). Figure 2 depicts our results for the dynamics of the averaged probability of double occupancy, $\left\langle P^{2}\right\rangle=\frac{1}{2} \sum_{i}\left\langle P_{i}^{2}\right\rangle$.

In Fig. 2(a), we present the evolution of a small system of six particles distributed on six sites, simulated with exact 

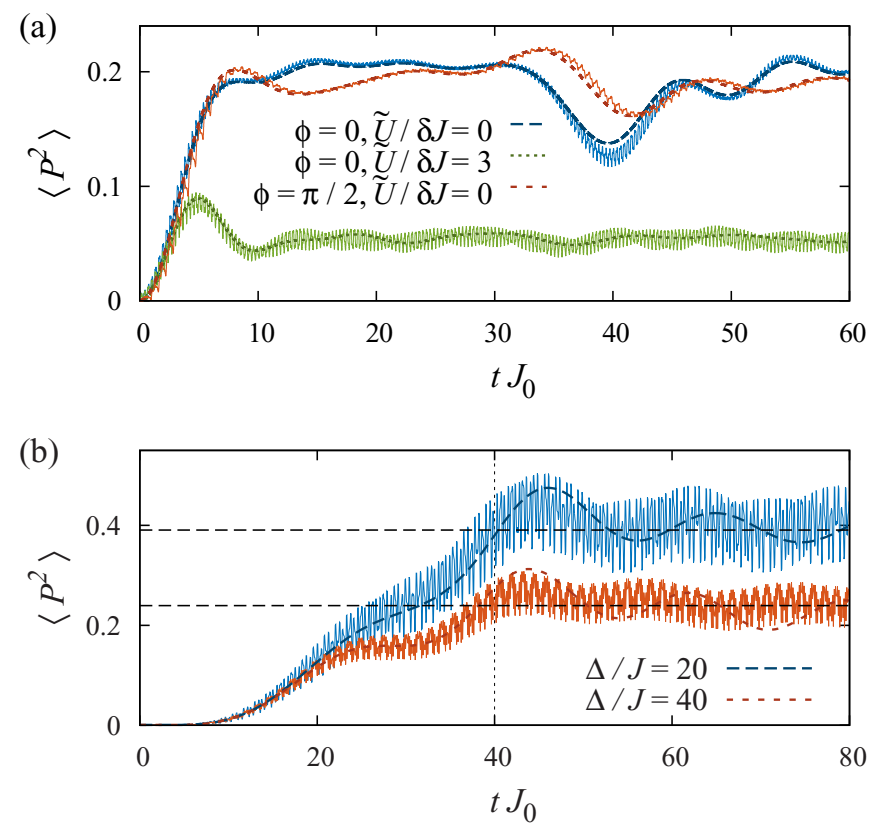

FIG. 2. Time evolution of the average double occupancy $\left\langle P^{2}\right\rangle$ : dashed (solid) curves represent the effective (full) model. (a) A small system is initially prepared in a Mott state with $\delta V=0$ and temperature $T=J_{0}$ (initial $\left\langle P^{2}\right\rangle \simeq 0$ ), and then it evolves after a sudden quench of $\delta V$, with $\Delta / J_{0}=40, U / J_{0}=20, \delta J_{1} / J_{0}=0.2$, and $\beta=1$, for different values of $\tilde{U} / \delta J_{1}$ and $\phi$. (b) Quasiadiabatic preparation (iTEBD results for $\rho=1$ ) of a ground state, starting from a MI and linearly increasing $\delta V(t)$ from zero to its final value in the time interval $0<J_{0} t<40$; we consider $U / J_{0}=5$, $\delta J_{1} / J_{0}=0.1, \tilde{U} / \delta J_{1}=2, \beta=1$. Remarkably, $\left\langle P^{2}\right\rangle(t)$ oscillates around the expected value (horizontal lines) for the ground state of the effective Hamiltonian with $\delta J$ corresponding to the final $\delta V$.

diagonalization methods. We initially set $\delta V=0$ and prepare a Mott-insulator (MI) state at $U \gg J_{0}$, assuming an initial temperature $T=J_{0}$, and hence negligible initial double occupancy $\left\langle P^{2}\right\rangle \simeq 0$. At time $t=0$ we abruptly turn on the modulation $\delta J(t)$. The results show a very good agreement between the effective model $\mathcal{H}_{\text {eff }}+\mathcal{H}_{\mathrm{NN}}$ and the full model of Eq. (1). Figure 2(a) shows that nonequilibrium experiments should be able to reveal both the ODG and the suppression of $\left\langle P^{2}\right\rangle$ resulting from the repulsive effective on-site interactions $\tilde{U}$. Similar agreement between the dynamics of the full and the effective model is obtained in the thermodynamic limit employing infinite time evolving block decimation (iTEBD) simulations [36], which are possible using the translational invariant formulation of Eq. (2).

The analysis of ground-state properties requires a (quasi-)adiabatic ramping of $\delta V$. We present in Fig. 2 our results obtained in the thermodynamic limit, using iTEBD. Starting again with $\delta V=0$ from an initial MI state, we studied the quasiadiabatic preparation of different MI ground states of our effective Hamiltonian with second-order corrections, for $\Delta=20$ and 40 (see Fig. 6 for ground-state phases). For computational simplicity we restrict the initially prepared state to $m=40$ matrix states, limiting its entanglement. During the time interval $0<t<t_{\text {ramp }}$ we linearly increase $\delta V$ to its final value, monitoring $\left\langle P^{2}\right\rangle$. Again $\mathcal{H}_{\text {eff }}+\mathcal{H}_{\mathrm{NN}}$ reproduces very well the dynamics of the full model (1). After the ramp, the heating induced by the quasiadiabatic character of the finite ramping time results in oscillations of $\left\langle P^{2}\right\rangle$ around the value expected for the ground state of the effective model.

\section{PHASES OF THE EFFECTIVE HAMILTONIAN}

At this point we focus on the ground-state physics of $\mathcal{H}_{\text {eff }}$, at first assuming that $J_{0} \ll \Delta,|\Delta \pm U|$, and hence that the intersite interaction $\mathcal{H}_{\mathrm{NN}}$ may be neglected, recovering it when it occurs to analyze how it affects the phase diagram. For $\beta \neq 1, \mathcal{H}_{\text {eff }}$ realizes a broad class of Hubbard models with correlated hopping extensively studied in the context of cuprate superconductors [37-41], and recently revisited for ultracold gases with modulated interactions [16,17]. For $\phi \neq 0$, the ODG gives rise to a particularly intriguing physics.

\section{A. Metal-insulator phase diagram}

We comment hereafter on Fig. 3, which shows, as a function of $\beta$ and the chemical potential $\mu$, the ground-state phase diagram of Fig. 8, in the limit of hard-core bosons $\phi=\pi / 2$, with balanced population of the two components, and for $\tilde{U}=0$. The result is obtained by means of density-matrix renormalization-group (DMRG) [42] simulations, in finitesize open-boundary systems of up to 80 sites, keeping up to 600 matrices. The Hamiltonian in Eq. (3) is symmetric under particle-hole exchange, and hence the region $\mu>0(\rho>1)$ is mirror symmetric to that depicted for $\mu<0(\rho<1)$ in Fig. 3(a).

We refer henceforth only to the range $\mu<0$. The case $\beta=0$ deserves particular attention. In this limit, the hopping processes (ii) and (iii) are not activated and doubly occupied sites (doublons) and empty ones (holons) become mutually impenetrable. The ground state is a metal without doublons (holons, for $\mu>0$ ), that tends to a lattice half filling $\rho=$ $1 / 2$ for $\mu \rightarrow 0$ (quarter filling of the energy band for each component). If existing, two doublons confine a localized metallic region within their sites, due to the doublon-holon mutual impenetrability; this results in a nonconducting metal with a vanishing Drude weight (Kohn metal) [38] for lattice fillings within $\rho=1 / 2$ and $3 / 2$ : all these states lie in the point of infinite compressibility $\mu=0$.

For $0<\beta<1$, in the absence of the ODG [17], the system undergoes a smooth phase transition from a metal (M) with dominant spin-density wave (SDW) correlations, $(-1)^{j}\left\langle n_{0-} n_{j-}\right\rangle$, with $n_{j-}=n_{j, \uparrow}-n_{j, \downarrow}, \quad$ to a triplet superconductor. In contrast, for $\phi=\pi / 2$, the metallic phase undergoes for $\beta \lesssim 1.4$ a commensurate-incommensurate phase transition, marked by a kink in the $\mu(\rho)$ curve, Fig. 3(b), to a peculiar gapless multicomponent (MC) phase. The MC phase presents a central charge $c \approx 3$ [43-45]; in contrast, the metallic phase has $c=2$. The MC phase smoothly connects to the Kohn metal for $\beta \rightarrow 0$.

For $\beta \gtrsim 1.4$ and $\rho \neq 1$, a spin gap $\Delta_{S}$ opens and the kink in $\mu(\rho)$ disappears, marking the transition to a phase with dominant singlet-superconducting (SS) correlations, $\left\langle\mathcal{Q}_{0-}^{\dagger} \mathcal{Q}_{j-}\right\rangle$, with $\mathcal{Q}_{j-} \equiv c_{j+1, \downarrow} c_{j, \uparrow}-c_{j+1, \uparrow} c_{j, \downarrow}$. Finally, at $\rho=1$ we find a MI with dominant SDW correlations, and a totally gapped phase with bond-ordering wave (BOW) order 

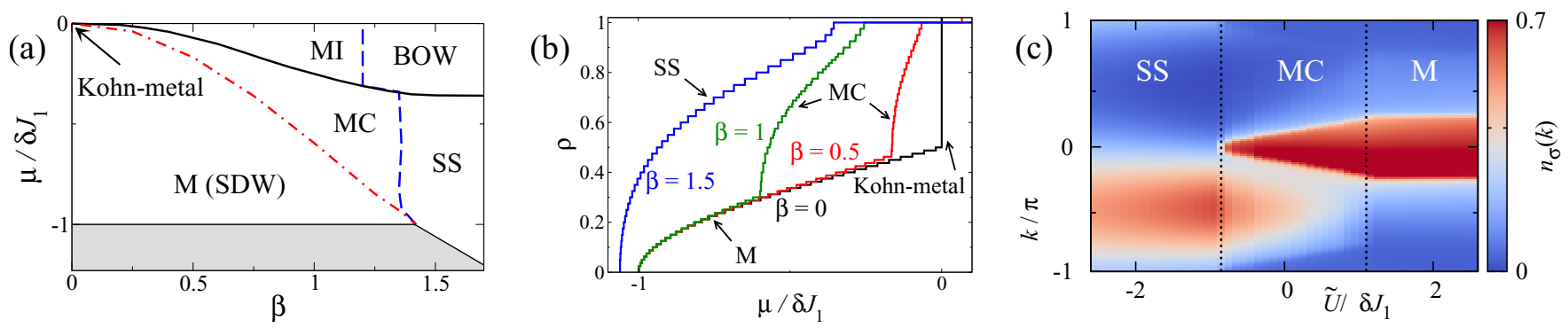

FIG. 3. (a) Phase diagram of $\mathcal{H}_{\text {eff }}$ as a function of $\mu / \delta J_{1}$ and $\beta$ for $\phi=\pi / 2$ and $\tilde{U}=0$. The dash-dotted lines mark the commensurateincommensurate metal-MC transition. The dashed (blue) lines denote the opening of $\Delta_{S}$ that marks the MC-SS and MI-BOW transitions. Shaded regions depict the vacuum. (b) Equation of state $\rho=\rho(\mu)$ for $\beta=0,0.5,1$, and 1.5 for the parameters of Fig. (a). (c) Single-component momentum distribution of the ground state of the effective Hamiltonian, Eq. (8), as a function of the effective onsite interaction $\tilde{U}$, for $\rho=0.5$ and $\phi=\pi / 2(L=60)$.

$O_{D}(j)=\sum_{\sigma} T_{\sigma}(j)-T_{\sigma}(j+1)$, with $T_{\sigma}(j)=c_{j, \sigma}^{\dagger} c_{j+1, \sigma}+$ H.c. and $O_{D}=\sum_{j} O_{D}(j)$.

\section{B. Multicomponent phase}

The multicomponent phase, which occurs even for $\beta=1$ and $\tilde{U}=0$, is a direct consequence of the ODG. The nature of this phase is best understood for $\phi=\pi / 2$ and $\beta=1$. In that case, the two-particle problem, with a $\uparrow$ particle and a $\downarrow$ one, presents for any $\tilde{U}$ an exact bound eigenstate:

$$
|P\rangle=\cos \theta|D\rangle+i \sin \theta|S\rangle,
$$

with eigenenergy

$$
E_{P}=\frac{\tilde{U}}{2}-\sqrt{\frac{\tilde{U}^{2}}{4}+2 \delta J_{1}^{2}}
$$

where

$$
\begin{aligned}
\tan \theta & =\frac{\tilde{U}-E_{P}}{\sqrt{2} \delta J_{1}}, \\
|D\rangle & =\sum_{j}(-1)^{j}|\uparrow, \downarrow\rangle_{j}, \\
|S\rangle & =\sum_{j}(-1)^{j}\left(|\uparrow\rangle_{j}|\downarrow\rangle_{j+1}-|\downarrow\rangle_{j}|\uparrow\rangle_{j+1}\right) / \sqrt{2}
\end{aligned}
$$

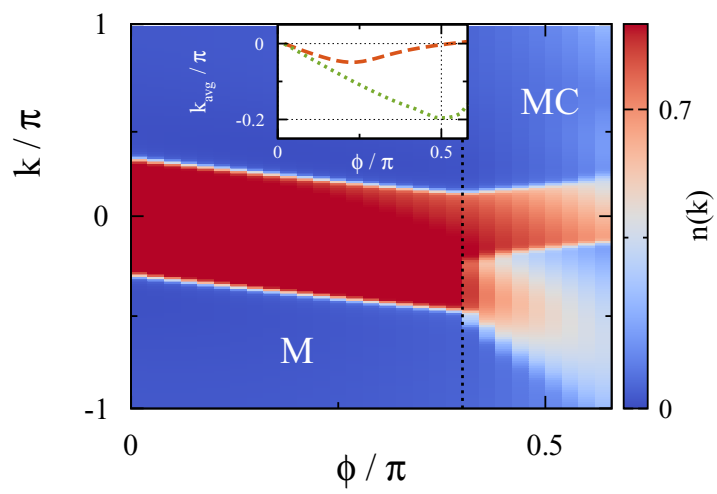

FIG. 4. Momentum distribution $n(k)$ of one component as a function of the Peierls phase. We used DMRG with $L=80$, filling $\rho=0.6, \tilde{U}=0$, and $\delta J=0.4$. The inset shows the center of the momentum distribution as a function of $\phi$ for different fillings $\rho=0.6$ (dashed line) and $\rho=0.2$ (dotted line). We included NN interactions, with $J=1, \Delta=10, U=20$.
The existence of this bound state even for $\tilde{U}>0$ results from the ODG (see Appendix A1). For sufficiently large $\tilde{U}>0$, the eigenenergy $E_{P}$ is larger than $E_{F}$ (the Fermi energy of the metal) and the metallic phase is stable. For decreasing $\tilde{U}$, the system enters the regime where $E_{P}<2 E_{F}$ and part of the Fermi sea forms pairs that quasicondense in $|P\rangle$, until the new Fermi energy $E_{F}^{\prime}=E_{P} / 2$. The $\mathrm{MC}$ phase results from the coexistence of a partially depleted Fermi sea and bound pairs. When $E_{F}^{\prime}$ reaches the bottom of the lattice band, the Fermi sea is fully depleted, marking the onset of the SS phase.

The MC phase has a characteristic momentum distribution of both components, $n_{\sigma}(k)$, and it can be thus easily revealed in time-of-flight measurements. The momentum distribution as a function of $\tilde{U} / \delta J$ is conveniently analyzed in the simple case $\beta=1$, for which the hopping processes share the same hopping rate $\delta J_{1} / 2$ :

$$
\mathcal{H}_{\text {eff }}=-\frac{\delta J_{1}}{2} \sum_{j, \sigma} c_{j+1, \sigma}^{\dagger} \mathrm{e}^{i \phi\left|n_{j+1, \bar{\sigma}}-n_{\bar{\sigma}, j}\right|} c_{j, \sigma}+\tilde{U} \mathcal{H}_{\text {int }} .
$$

Figure 3(c) shows our results for $n_{\sigma}(k)$, at $\phi=\pi / 2$. For large-enough $\tilde{U}$, the metallic phase presents a slablike Fermi sea. In the MC phase, the slab shrinks due to partial pairing. The latter results in a blurred contribution to $n_{\sigma}(k)$, $\frac{1}{2 \pi}\left[1-\sqrt{2} \sin (2 \theta) \sin (k / 2)-\sin ^{2} \theta \cos (2 k)\right]$, as expected for $|P\rangle$ pairs (see Appendix A2). The MC-SS transition is marked by the vanishing Fermi sea.

Lastly, we show in Fig. 4 how the MC phase enters the system as the Peierls phase increases, presenting numerical results on the momentum distribution. We set $\rho=0.6, \tilde{U}=0$, and $\delta J_{1}=0.4$; up to a threshold Peierls phase, we observe a metallic phase with drifted momentum distribution, and beyond it the momentum distribution splits into a blurred lower branch corresponding to the bound pairs, and a more definite upper branch, the metallic component. The center of the momentum distribution $k_{\mathrm{avg}}$, in the inset, has a maximum in the drift which is more pronounced and occurs at larger phases when the lattice filling is smaller.

\section{Two-component anyon-Hubbard model}

At low lattice filling $\rho$, for which processes (iv) may be neglected, a Jordan-Wigner like transformation [20],

$$
f_{j, \sigma}=e^{-i 2 \phi \sum_{1 \leqslant l<j} n_{l}} e^{-i \phi n_{j}} c_{j, \sigma},
$$




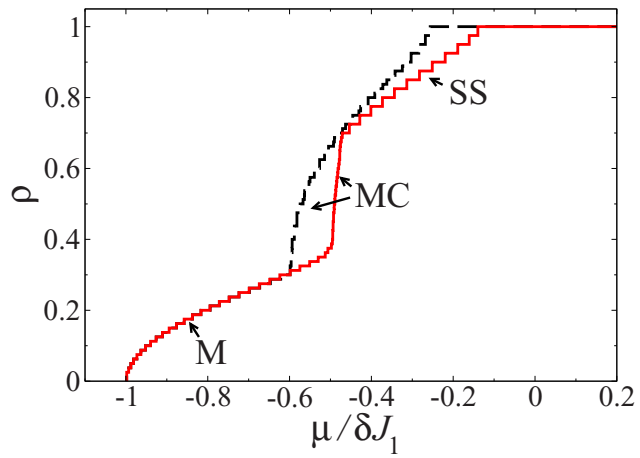

FIG. 5. Comparison between the effective Hamiltonian of Eq. (8) and the anyon-Hubbard model of Eq. (10), for $\tilde{U}=0, \phi=\pi / 2$, and $\beta=1$.

maps Eq. (8) into a two-component anyon-Hubbard model (2-AHM):

$$
\mathcal{H}_{\mathrm{AHM}}=-\frac{\delta J_{1}}{2} \sum_{j, \sigma}\left(f_{j+1, \sigma}^{\dagger} f_{j, \sigma}+\text { H.c. }\right)+\tilde{U} \mathcal{H}_{\text {int }} .
$$

The operators $f_{j, \sigma}$ and $f_{j, \sigma}^{\dagger}$ characterize anyonlike hardcore particles, that fulfill a deformed exchange statistics (DES):

$$
\begin{aligned}
f_{j, \sigma} f_{k, \sigma^{\prime}}^{\dagger}+\mathcal{F}_{j, k} f_{k, \sigma^{\prime}}^{\dagger} f_{j, \sigma} & =\delta_{j, k} \delta_{\sigma, \sigma^{\prime}}, \\
f_{j, \sigma} f_{k, \sigma^{\prime}}+\mathcal{F}_{j, k} f_{k, \sigma^{\prime}} f_{j, \sigma} & =0 .
\end{aligned}
$$

The $\mathbb{C}$ parameter $\mathcal{F}_{j, k}$ determines the statistics of the system:

$$
\mathcal{F}_{j, k}:=\left\{\begin{array}{ll}
e^{-i 2 \phi}, & j>k \\
1, & j=k \\
e^{i 2 \phi}, & j<k
\end{array},\right.
$$

where the condition $\mathcal{F}_{j, j}=1$ sets the hard-core behavior of the particles. For $\phi=0$ one recovers the standard two-component Fermi-Hubbard model, while $\phi=\pi / 2$ corresponds to the two-component hard-core Bose-Hubbard model. In Fig. 5 we present a comparison between the phase diagram of the effective Hamiltonian of Eq. (8), with $\beta=1$, and the anyon-Hubbard model of Eq. (10), for $\tilde{U}=0$ and $\phi=\pi / 2$. Both models provide identical results at low densities. Not unexpectedly they diverge when the density grows $(\rho>0.3)$. As for the effective model, the anyon model shows as well a (narrower) MC phase. At larger densities close to $\rho=1$ model (10) presents an intermediate SS phase absent in the effective Hamiltonian (8).

Specific cases of the 2-AHM have been studied in the context of exactly solvable models [46,47]. In contrast, the nonintegrable DES discussed here does strongly modify the spectrum of the 2-AHM compared to the Fermi-Hubbard model.

\section{Intersite interactions}

The nearest-neighbor interaction $\mathcal{H}_{\mathrm{NN}}$ becomes relevant for large-enough $J_{0} / \Delta, J_{0} /|\Delta \pm U|$. Combining effective onsite and NN interactions constitutes an additional interesting control possibility resulting from the three-color modulation.

Figure 6 depicts for $\beta=1, \phi=0$, and $\rho=1$ the phase diagram as a function of $\tilde{U} / \delta J$ and $J_{0} / \Delta$ (which controls

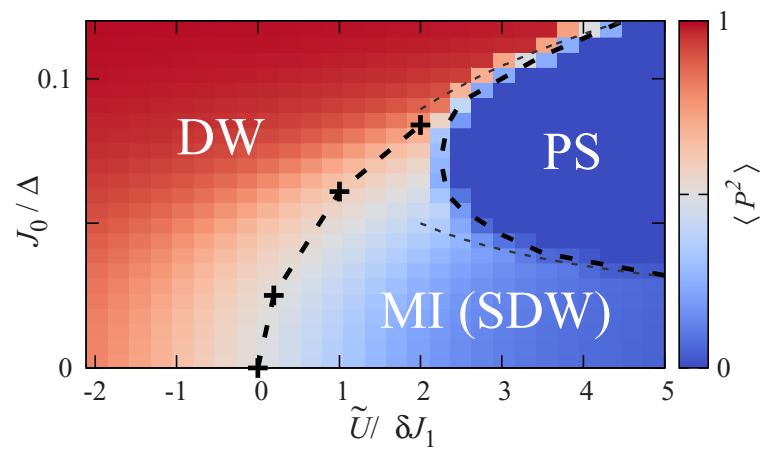

FIG. 6. Phase diagram of $\mathcal{H}_{\text {eff }}+\mathcal{H}_{\mathrm{NN}}$ for $\rho=1, U=5 J_{0}, \delta J=$ $0.1 J_{0}, \beta=1$, and $\phi=0$. The MI-DW transition is given by $K_{S}=1$ (extrapolated from DMRG calculations of up to $L=160$ sites). The coloring codes $\left\langle P^{2}\right\rangle$ are obtained from iTEBD calculations (for 200 states results are consistent with our DMRG data of 160 sites).

the strength of the NN terms). For $J_{0} / \Delta \rightarrow 0$ the standard two-component 1D Fermi-Hubbard model is recovered [48]: for any $\tilde{U}>0$ there is a Mott-insulator (SDW) phase with a finite charge excitation gap $\Delta_{c}>0$ and spin gap null $\Delta_{S}=0$; in contrast, for $\tilde{U}<0$, a spin gap $\Delta_{S}>0$ opens, while $\Delta_{c}=0$. For sufficiently large $J_{0} / \Delta$ the system is driven into a fully gapped density-wave (DW) phase, characterized by a nonvanishing DW order parameter $O_{\mathrm{DW}}=\sum_{j}(-1)^{j}\left\langle n_{0} n_{j}\right\rangle$. For a dominant intersite interaction, the first term of Eq. (4) favors the crystalline phase with-neglecting quantum fluctuationsan energy

$$
E_{\mathrm{DW}} \sim \tilde{U}-\frac{4 U}{\Delta^{2}-U^{2}} .
$$

In this region, the condition $E_{\mathrm{DW}}=0$ gives a rough estimate of the DW phase boundary, which also marks the transition between the DW and the PS phase (dotted upper line). For $\tilde{U}>0$ we observe two different MI phases with a suppressed doublon number: the above-mentioned MI phase with antiferromagnetically ordered spin, that means dominant SDW correlations and a vanishing spin gap $\Delta_{S}=0$, and a region of phase separation of ferromagnetic domains (PS). The interplay between the two phases can be understood best from an expansion in the limit of strong interaction $\tilde{U} \gg \delta J$. In this limit, one can project out the doubly occupied sites and obtain an effective spin-1/2 model with superexchange interaction $\mathcal{H}_{\mathrm{se}} \sim J_{\mathrm{eff}} \vec{S} \cdot \vec{S}$, with

$$
J_{\mathrm{eff}}=\frac{2 U}{\delta^{2}-U^{2}}-\frac{\delta J^{2}}{4}\left(\frac{1}{\tilde{U}+\frac{2 U}{U \Delta-\Delta^{2}}}+\frac{1}{\tilde{U}+\frac{2 U}{U \Delta+\Delta^{2}}}\right) .
$$

At $1 / \Delta \sim \delta J / \sqrt{4 U \tilde{U}}, J_{\text {eff }}$ changes sign and this marks the transition from the SDW phase $\left(J_{\text {eff }}>0\right)$ to the PS region $\left(J_{\text {eff }}<0\right)$, as shown in Fig. 6 with a lower dashed line. In our DMRG and iTEBD calculations we could clearly characterize the first-order transition to the PS region by a marked drop of the doublon density, as illustrated by the color distribution in the figure.

The MI-DW transition is associated to the opening of the spin gap $\Delta_{S}$, characterized by the Luttinger-liquid parameter in the spin sector $K_{S}=1$ ("+" symbols in Fig. 6). We extract $K_{S}$ 


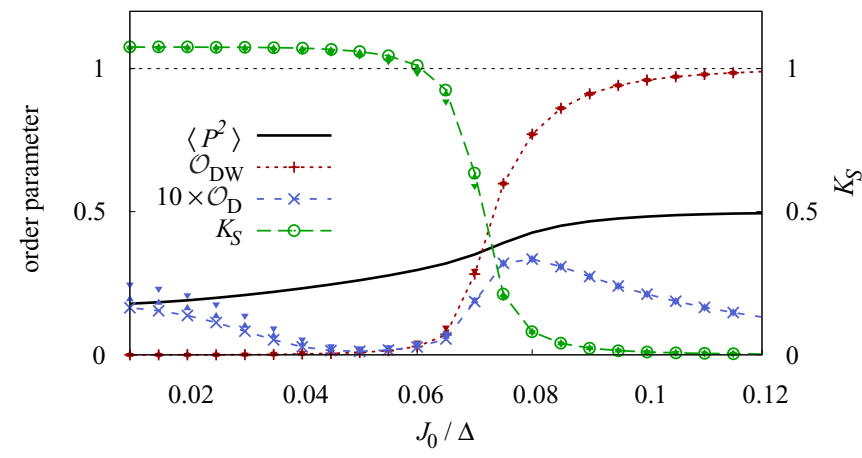

FIG. 7. Average doublon density $\left\langle P^{2}\right\rangle$, order parameters $O_{\mathrm{DW}}$ and $O_{D}$, and Luttinger-liquid parameter $K_{S}$ (cut of Fig. 6 at $\tilde{U} / \delta J=1$ ): DMRG simulations with $L=180$ sites; small filled downwards- and upwards-pointing triangles depict results for $L=80$ and 120 sites).

from the long-wavelength behavior of the static spin structure factor [49]:

$$
S_{n_{-}}(k)=\frac{1}{L} \sum_{i, j} e^{i(i-j) k}\left\langle n_{i-} n_{j-}\right\rangle .
$$

Since $\mathcal{H}_{\mathrm{NN}}$ breaks the spatial reflection symmetry, we do not observe a separate BOW phase, as it is the case for Hubbard models with standard density-density NN interactions [50], but a nonvanishing BOW order $O_{D}$ in the DW phase, due to the preferred creation of excitations in a particular spatial direction.

Several physical quantities and order parameters have been considered and numerically calculated in order to mark the phases and the phase transitions described so far. Figure 7 presents a plot of them on a vertical cut of the phase diagram of Fig. 6: we fix $\tilde{U} / \delta J_{1}=1$ and vary $\Delta$ across the MI to DW phase transition. As already evident from the color scale in Fig. 6, the average double occupancy $\left\langle P^{2}\right\rangle$ increases rather smoothly from a finite value at zero $\mathrm{NN}$ interaction to the maximum $\left\langle P^{2}\right\rangle=0.5$ in the deep density-wave phase limit, already at $J_{0} / \Delta=0.12$. In the same range, the DW order parameter ranges from zero in the MI state to its maximum value on a narrower interval, which allows us to mark more precisely the MI-DW transition. Conversely, the Luttingerparameter clearly characterizes the opening of a spin gap $\Delta_{S}$ and the simultaneous suppression of SDW correlations around the region $J_{0} / \Delta \simeq 0.6$ and 0.8 . The BOW order is instead completely enclosed in the dominating DW curve, which does not allow us to distinguish uniquely the BOW phase.

\section{CONCLUSION AND OUTLOOK}

A multicolor modulation of the lattice depth allows for a flexible separate manipulation of (a) correlated hopping, controlled by the modulation amplitudes $\delta V_{s}$; (b) occupationdependent gauge fields, given by the phase shift $\phi_{s}$; (c) effective on-site interactions, provided by the detuning $\tilde{U}$; and (d) NN interactions, that depend on $J_{0} / \Delta$ and $J_{0} /|\Delta \pm U|$. The three-color modulation thus provides an experimentally straightforward method for engineering a very broad class of lattice models, including Hubbard Hamiltonians with correlated hopping, peculiar extended models, and the twocomponent anyon-Hubbard model. In particular, the controllable quantum statistics of the 2-AHM results in a peculiar multicomponent phase of coexisting superconducting and metallic components.

Multicolor modulation permits several further interesting extensions, including the control of three-body interactions [51]. In combination with a Raman-induced coupling of several spin components [11,12] one may study density dependent magnetic fields [31]. Other scenarios could pave a realistic exploration path towards the simulation of dynamical gauge fields with cold atoms in optical lattices, exploring, e.g., occupation-dependent non-Abelian fields and gauge fields in Fermi-Bose mixtures.

\section{ACKNOWLEDGMENTS}

We thank L. Fallani, A. Eckardt, and C. Morais Smith for discussions. We acknowledge the support of QUEST-LFS and the Deutsche Forschungsgemeinschaft Research Training Group 1729. Simulations were carried out on the cluster system at the Leibniz University of Hannover, Germany.

\section{APPENDIX A: MULTICOMPONENT PHASE}

\section{Two-particle model}

We assume for simplicity $\beta=1$, hence we consider the model described in Eq. (8). We are interested in the twoparticle problem, with one $\uparrow$ particle and one $\downarrow$ particle. Let $|D(j)\rangle$ be a doubly occupied site, and $|S(j, j+l)\rangle$ a singlet state placed in sites $j$ and $j+l$. Then

$$
\begin{gathered}
\mathcal{H}_{\mathrm{eff}}|D(j)\rangle=-\frac{\delta J_{1}}{\sqrt{2}}\left[e^{i \phi}|S(j, j+1)\rangle+e^{-i \phi}|S(j-1, j)\rangle\right]+\tilde{U}|D(j)\rangle \\
\mathcal{H}_{\mathrm{eff}}|S(j, j+1)\rangle=-\frac{\delta J_{1}}{\sqrt{2}}\left[e^{i \phi}|D(j+1)\rangle+e^{-i \phi}|D(j)\rangle\right]-\frac{\delta J_{1}}{2}[|S(j-1, j+1)\rangle+|S(j, j+2)\rangle], \\
\mathcal{H}_{\mathrm{eff}}|S(j, j+l)\rangle \stackrel{l>1}{=}-\frac{\delta J_{1}}{2}[|S(j-1, j+l)\rangle+|S(j+1, j+l)\rangle+|S(j, j+l-1)\rangle+|S(j, j+l+1)\rangle]
\end{gathered}
$$

Let us define

$$
\begin{gathered}
|D(k)\rangle=\frac{1}{\sqrt{L}} \sum_{l} e^{i k l}|D(l)\rangle, \\
|S(j, k)\rangle=\frac{1}{\sqrt{L}} \sum_{l} e^{i k(l+j / 2)}|S(l, l+j)\rangle
\end{gathered}
$$


with $k$ the center-of-mass momentum of the pair, and $L$ the number of sites. Then $\mathcal{H}_{\text {eff }}=\sum_{k} \mathcal{H}_{\text {eff }}(k)$, with $\mathcal{H}_{\text {eff }}(k)=$ $\mathcal{H}_{0}(k)+\mathcal{H}_{1}(k)$, where

$$
\begin{aligned}
& \mathcal{H}_{0}(k)=\tilde{U}|D(k)\rangle\langle D(k)|-A(k)[|S(1, k)\rangle\langle D(k)|+\text { H.c. }], \\
& \mathcal{H}_{1}(k)=-B(k) \sum_{j \geqslant 1}[|S(j, k)\rangle\langle S(j+1, k)|+\text { H.c. }],
\end{aligned}
$$

with $A(k)=\sqrt{2} \delta J_{1} \cos (k / 2-\phi)$ and $B(k)=\delta J_{1} \cos (k / 2)$. We may diagonalize $\mathcal{H}_{0}$ :

$$
\mathcal{H}_{0}(k)=E_{+}(k)|\tilde{P}(k)\rangle\left\langle\tilde{P}(k)\left|+E_{-}(k)\right| P(k)\right\rangle\langle P(k)|,
$$

where the eigenenergies are

$$
E_{ \pm}(k)=\frac{\tilde{U}}{2} \pm \sqrt{\left(\frac{\tilde{U}}{2}\right)^{2}+A(k)^{2}},
$$

and the corresponding eigenstates are

$$
\begin{aligned}
& |\tilde{P}(k)\rangle=\cos \theta(k)|D(k)\rangle+\sin \theta(k)|S(1, k)\rangle, \\
& |P(k)\rangle=-\sin \theta(k)|D(k)\rangle+\cos \theta(k)|S(1, k)\rangle,
\end{aligned}
$$

with $\tan \theta(k)=\frac{\tilde{U} / 2-E_{-}(k)}{A(k)}$. The Hamiltonian $\mathcal{H}_{0}$ characterizes deeply bound pairs. We may then split $\mathcal{H}_{1}(k)=\mathcal{H}_{c}(k)+$ $\mathcal{H}_{u}(k)$, where

$$
\mathcal{H}_{u}(k)=-B(k) \sum_{j \geqslant 2}[|S(j, k)\rangle\langle S(j+1, k)|+\text { H.c. }]
$$

determines the physics of broken pairs, where the dynamics of the relative coordinate $j$ is given by the hopping rate $B(k)$, and

$$
\begin{aligned}
\mathcal{H}_{c}(k)= & -B(k)(\sin \theta(k)|P(k)\rangle \\
& +\cos \theta(k)|\tilde{P}(k)\rangle)\langle S(2, k)|+\text { H.c. }
\end{aligned}
$$

characterizes the coupling between deeply bound and unbound pairs. Note that such a coupling is also given by $B(k)$.

Let us consider $\phi=\frac{\pi}{2}$. In that case,

$$
E_{ \pm}(k)=\frac{\tilde{U}}{2} \pm \sqrt{\left(\frac{\tilde{U}}{2}\right)^{2}+2 \delta J_{1}^{2} \sin ^{2}(k / 2)} .
$$

The minimal energy is clearly for $k=\pi, E_{P} \equiv$ $E_{-}(\pi)=\frac{\tilde{U}}{2} \pm \sqrt{\left(\frac{\tilde{U}}{2}\right)^{2}+2 \delta J_{1}^{2}}$. If existing, bound pairs will quasicondense in $|P\rangle \equiv|P(\pi)\rangle$. Crucially, $B(\pi)=0$, and hence $\mathcal{H}_{c}=0$. As a result, $|P\rangle$ remains a deeply bound two-particle eigenstate, fully decoupled from the unbound pairs, irrespective of the value of $\tilde{U} / \delta J_{1}$. In contrast, for $\phi=0$, i.e., without the ODG, the bound pairs are fully connected with the rest and cannot be formed unless $\tilde{U}<0$ dominates. For $\phi$ in the vicinity of $\pi / 2$ the coupling $\mathcal{H}_{c}$ may be considered perturbative, and deeply bound pairs due to the ODG still exist even if $\phi$ is not exactly $\pi / 2$.

The existence of these pairs that are deeply bound by the ODG rather than by attractive interactions is crucial to understand the nature of the MC phase. The metallic phase is stable if $E_{P} / 2>E_{F}$, with $E_{F}$ the Fermi energy of the metal. However, for decreasing $\tilde{U}>0, E_{F}<E_{P} / 2$, and hence it is energetically favorable to pair part of the Fermi sea into $|P\rangle$ pairs, until reaching an equilibrium at a new Fermi energy $E_{F}^{\prime}=E_{P} / 2$. This partial pairing, and the corresponding coexistence of a two-component metal and a superconductor, explains the MC phase and its $c=3$ central charge. For $E_{-}(\pi)<-2 \delta J_{1}$ (which occurs at $\tilde{U} / \delta J_{1} \simeq-1$ ) the Fermi sea is completely depleted, and the system enters the fully paired (SS) phase.

\section{Momentum distribution}

The momentum distribution of the $\uparrow$ component in the $|P\rangle$ state is $n_{\uparrow}^{(P)}(k)=\sum_{i, j} e^{i k(i-j)}\left\langle P\left|c_{i, \uparrow}^{\dagger} c_{j, \uparrow}\right| P\right\rangle$, where

$$
\begin{gathered}
\left\langle P\left|c_{l, \uparrow}^{\dagger} c_{l, \uparrow}\right| P\right\rangle=\frac{1}{L}, \\
\left\langle P\left|c_{l+1, \uparrow}^{\dagger} c_{l, \uparrow}\right| P\right\rangle=\left\langle P\left|c_{l-1, \uparrow}^{\dagger} c_{l, \uparrow}\right| P\right\rangle^{*}=\frac{-\sin [2 \theta(\pi)]}{L \sqrt{2}} e^{i \pi / 2},
\end{gathered}
$$

$\left\langle P\left|c_{l+2, \uparrow}^{\dagger} c_{l, \uparrow}\right| P\right\rangle=\left\langle P\left|c_{l-2, \uparrow}^{\dagger} c_{l, \uparrow}\right| P\right\rangle=\frac{-\sin ^{2}[\theta(\pi)]}{2 L}$,

and other correlations are zero. After normalizing,

$$
\begin{aligned}
n_{\uparrow}^{(P)}(k)= & \frac{1}{2 \pi}[1-\sqrt{2} \sin [2 \theta(\pi)] \sin (k / 2) \\
& \left.-\sin ^{2} \theta(\pi) \cos (2 k)\right]
\end{aligned}
$$

with $\theta(\pi)=\arctan \left[\chi+\sqrt{\chi^{2}+1}\right]$, with $\chi=\frac{\tilde{U}}{2 \sqrt{2} \delta J_{1}}$. For the $\downarrow$ component the expression is identical. This expression is in excellent agreement with the blurred momentum distribution that is found in our numerics in the MC phase [Fig. 3(c)] in addition to the partially depleted slablike Fermi sea.

\section{APPENDIX B: DERIVATION OF THE EFFECTIVE MODEL VIA MAGNUS EXPANSION}

For the simplified case of a time periodic Hamiltonian, i.e., assuming that the frequencies $\omega_{1}=\Delta$ and $\omega_{2}=\Delta+U$ are integer multiples of $\omega=|\Delta-U|\left(\omega_{1}=m_{1} \omega, \omega_{2}=m_{2} \omega\right)$, we may obtain the same effective Hamiltonian of Eqs. (3) and (4) employing a formal Magnus expansion [52-55] or Floquet analysis [22]. Following the presentation of Ref. [55] we may express the effective Hamiltonian as a series in $1 / \omega$ as $\mathcal{H}_{\text {eff }}=\mathcal{H}^{(0)}+\mathcal{H}_{M E}^{(1)}+O\left(\frac{1}{\omega^{2}}\right)$, ultimately resulting from an integral over time on period $T=2 \pi / \omega$. The lowest-order term

$$
\mathcal{H}^{(0)}=\frac{1}{T} \int_{0}^{T} d t_{1} \mathcal{H}\left(t_{1}\right)
$$

provides Eq. (3). The first-order correction in $1 / \omega$ may be expressed as [52]

$$
\mathcal{H}_{M E}^{(1)}=\frac{-i}{2 T} \int_{0}^{T} d t_{2} \int_{0}^{t_{2}} d t_{1}\left[\mathcal{H}\left(t_{2}\right), \mathcal{H}\left(t_{1}\right)\right]
$$


If the time periodic Hamiltonian is given by a Fourier series $\mathcal{H}(t)=\mathcal{H}_{0}+\sum_{n} V^{(n)} e^{i n \omega t}$, then

$$
\mathcal{H}_{\mathrm{ME}}^{(1)}=\frac{1}{\omega} \sum_{n} \frac{1}{n}\left(\left[V^{(n)}, V^{(-n)}\right]-\left[V^{(n)}, \mathcal{H}_{0}\right]+\left[V^{(-n)}, \mathcal{H}_{0}\right]\right)
$$

In our case, the Fourier series is restricted to the following three terms:

$$
V^{( \pm 1)}=-\frac{\delta J_{3}}{2} \mathcal{H}_{\text {hop }}, \quad V^{\left( \pm m_{1}\right)}=-e^{ \pm i \phi} \frac{\delta J_{1}}{2} \mathcal{H}_{\text {hop }}, \quad V^{\left( \pm m_{2}\right)}=-e^{ \pm i \phi} \frac{\delta J_{2}}{2} \mathcal{H}_{\text {hop }},
$$

where $\mathcal{H}_{\text {hop }}=\sum_{j, \sigma} c_{j+1, \sigma}^{\dagger} c_{j, \sigma}$. Equation (2) can be recovered by expanding the exponential term $e^{ \pm i t U n_{j \sigma}}=1+\left(e^{ \pm i t U}-1\right) n_{j \sigma}$. Then

$$
\tilde{\mathcal{H}}(t)=\left[J_{0}+\delta J(t)\right]\left(e^{i t[\Delta-U]} \bar{V}^{(1)}+e^{i t \Delta} \bar{V}^{(2)}+e^{i t[\Delta+U]} \bar{V}^{(3)}+\text { H.c. }\right)
$$

with

$$
\bar{V}^{(1)}=\sum_{j, \sigma} d_{j, \sigma}^{\dagger} c_{j+1, \sigma}-d_{j, \sigma}^{\dagger} d_{j+1, \sigma}, \quad \bar{V}^{(2)}=\sum_{j, \sigma}\left(d_{j, \sigma}^{\dagger}-c_{j, \sigma}\right)\left(d_{j+1, \sigma}^{\dagger}-c_{j+1, \sigma}\right), \quad \bar{V}^{(3)}=\sum_{j, \sigma} c_{j, \sigma}^{\dagger} d_{j+1, \sigma}-d_{j, \sigma}^{\dagger} d_{j+1, \sigma} .
$$

where we employ the correlated annihilation operator $d_{j, \sigma} \equiv n_{j, \bar{\sigma}} c_{j, \sigma}$. Neglecting terms of order $J_{0} \delta J$ and $\delta J^{2}$ we may write

$$
\mathcal{H}_{\mathrm{ME}}^{(1)}=\frac{J_{0}^{2}}{\Delta-U}\left[\bar{V}^{(1)}, \bar{V}^{(1) \dagger}\right]+\frac{J_{0}^{2}}{\Delta}\left[\bar{V}^{(2)}, \bar{V}^{(2) \dagger}\right]+\frac{J_{0}^{2}}{\Delta+U}\left[\bar{V}^{(3)}, \bar{V}^{(3) \dagger}\right]+O(\delta J),
$$

which after some algebra yields Eq. (4).

[1] T. Dittrich, P. Hänggi, G.-L. Ingold, B. Kramer, G. Schön, and W. Zwerger, in Quantum Transport and Dissipation (Wiley-VCH, New York, 1998), Chap. 5.

[2] A. Eckardt, C. Weiss, and M. Holthaus, Phys. Rev. Lett. 95, 260404 (2005).

[3] H. Lignier, C. Sias, D. Ciampini, Y. Singh, A. Zenesini, O. Morsch, and E. Arimondo, Phys. Rev. Lett. 99, 220403 (2007).

[4] E. Kierig, U. Schnorrberger, A. Schietinger, J. Tomkovic, and M. K. Oberthaler, Phys. Rev. Lett. 100, 190405 (2008).

[5] A. Zenesini, H. Lignier, D. Ciampini, O. Morsch, and E. Arimondo, Phys. Rev. Lett. 102, 100403 (2009).

[6] J. Struck, C. Ölschläger, R. Le Targat, P. Soltan-Panahi, A. Eckardt, M. Lewenstein, P. Windpassinger, and K. Sengstock, Science 333, 996 (2011).

[7] J. Struck, C. Olschlager, M. Weinberg, P. Hauke, J. Simonet, A. Eckardt, M. Lewenstein, K. Sengstock, and P. Windpassinger, Phys. Rev. Lett. 108, 225304 (2012).

[8] C. V. Parker, L. C. Ha, and C. Chin, Nat. Phys. 9, 769 (2013).

[9] M. Aidelsburger, M. Atala, M. Lohse, J. T. Barreiro, B. Paredes, and I. Bloch, Phys. Rev. Lett. 111, 185301 (2013).

[10] H. Miyake, G. A. Siviloglou, C. J. Kennedy, W. C. Burton, and W. Ketterle, Phys. Rev. Lett. 111, 185302 (2013).

[11] M. Mancini et al., Science 349, 1510 (2015).

[12] B. K. Stuhl, H.-I. Lu, L. M. Aycock, D. Genkina, and I. B. Spielman, Science 349, 1514 (2015).

[13] J. Gong, L. Morales-Molina, and P. Hänggi, Phys. Rev. Lett. 103, 133002 (2009).

[14] F. K. Abdullaev, P. G. Kevrekidis, and M. Salerno, Phys. Rev. Lett. 105, 113901 (2010).

[15] Á. Rapp, X. Deng, and L. Santos, Phys. Rev. Lett. 109, 203005 (2012).

[16] M. Di Liberto, C. E. Creffield, G. I. Japaridze, and C. Morais Smith, Phys. Rev. A 89, 013624 (2014).

[17] S. Greschner, L. Santos, and D. Poletti, Phys. Rev. Lett. 113, 183002 (2014).
[18] F. Meinert, M. J. Mark, K. Lauber, A. J. Daley, and H.-C. Nägerl, Phys. Rev. Lett. 116, 205301 (2016).

[19] S. Greschner, G. Sun, D. Poletti, and L. Santos, Phys. Rev. Lett. 113, 215303 (2014).

[20] T. Keilmann, S. Lanzmich, I. McCulloch, and M. Roncaglia, Nat. Commun. 2, 361 (2011).

[21] S. Greschner and L. Santos, Phys. Rev. Lett. 115, 053002 (2015)

[22] C. Sträter, S. C. Srivastava, and A. Eckardt, arXiv:1602.08384.

[23] Y. Hao, Y. Zhang, and S. Chen, Phys. Rev. A 79, 043633 (2009).

[24] G. Tang, S. Eggert, and A. Pelster, New J. Phys. 17, 123016 (2015).

[25] A. del Campo, Phys. Rev. A 78, 045602 (2008).

[26] Y. Hao and S. Chen, Phys. Rev. A 86, 043631 (2012).

[27] L. Wang, L. Wang, and Y. Zhang, Phys. Rev. A 90, 063618 (2014).

[28] U. J. Wiese, Ann. Phys. 525, 777 (2013).

[29] A. Bermudez and D. Porras, New J. Phys. 17, 103021 (2015).

[30] M. J. Edmonds, M. Valiente, G. Juzeliunas, L. Santos, and P. Ohberg, Phys. Rev. Lett. 110, 085301 (2013).

[31] S. Greschner, D. Huerga, G. Sun, D. Poletti, and L. Santos, Phys. Rev. B 92, 115120 (2015).

[32] R. Ma, M. E. Tai, P. M. Preiss, W. S. Bakr, J. Simon, and M. Greiner, Phys. Rev. Lett. 107, 095301 (2011).

[33] W. Zwerger, J. Opt. B: Quantum Semiclass. Opt. 5, S9 (2003).

[34] See M. A. Cazalilla and A. M. Rey, Rep. Prog. Phys. 77, 124401 (2014), and references therein.

[35] M. Höfer, L. Riegger, F. Scazza, C. Hofrichter, D. R. Fernandes, M. M. Parish, J. Levinsen, I. Bloch, and S. Folling, Phys. Rev. Lett. 115, 265302 (2015).

[36] G. Vidal, Phys. Rev. Lett. 98, 070201 (2007).

[37] L. Arrachea and A. A. Aligia, Phys. Rev. Lett. 73, 2240 (1994).

[38] L. Arrachea, A. A. Aligia, and E. Gagliano, Phys. Rev. Lett. 76, 4396 (1996).

[39] L. Arrachea, E. R. Gagliano, and A. A. Aligia, Phys. Rev. B 55, 1173 (1997). 
[40] A. A. Aligia and L. Arrachea, Phys. Rev. B 60, 15332 (1999).

[41] A. A. Aligia, K. Hallberg, C. D. Batista, and G. Ortiz, Phys. Rev. B 61, 7883 (2000).

[42] U. Schollwöck, Ann. Phys. 326, 96 (2011).

[43] We evaluate the central charge from the conformal expression of the von-Neumann entropy, $S_{v N, L}(l)=\frac{c}{3} \ln \left[\frac{L}{\pi} \sin \left(\frac{\pi}{L} l\right)\right]+\gamma$, for a subsystem of length $l$ in a system of $L$ sites, with $\gamma$ a constant $[44,45]$.

[44] G. Vidal, J. I. Latorre, E. Rico, and A. Kitaev, Phys. Rev. Lett. 90, 227902 (2003).

[45] P. Calabrese and J. Cardy, J. Stat. Mech. (2004) P06002.

[46] H. J. Schulz and B. S. Shastry, Phys. Rev. Lett. 80, 1924 (1998).

[47] A. Osterloh, L. Amico, and U. Eckern, J. Phys. A 33, L87 (2000).
[48] F. H. Essler, H. Frahm, F. Göhmann, A. Klümper, and V. E. Korepin, The One-Dimensional Hubbard Model (Cambridge University Press, Cambridge, England, 2005).

[49] A. Moreno, A. Muramatsu, and S. R. Manmana, Phys. Rev. B 83, 205113 (2011).

[50] S. Ejima and S. Nishimoto, Phys. Rev. Lett. 99, 216403 (2007).

[51] A. J. Daley and J. Simon, Phys. Rev. A 89, 053619 (2014).

[52] M. M. Maricq, Phys. Rev. B 25, 6622 (1982).

[53] G. Jotzu, M. Messer, R. Desbuquois, M. Lebrat, T. Uehlinger, D. Greif, and T. Esslinger, Nature (London) 515, 237 (2014).

[54] N. Goldman and J. Dalibard, Phys. Rev. X 4, 031027 (2014).

[55] M. Aidelsburger, M. Lohse, C. Schweizer, M. Atala, J. T. Barreiro, S. Nascimbène, N. R. Cooper, I. Bloch, and N. Goldman, Nat. Phys. 11, 162 (2015). 